\title{
Haemophilus influenzae associated scleritis
}

Scott O Sykes, Christopher Riemann, Carmen I Santos, David M Meisler, Careen Y Lowder, John P Whitcher, Emmett T Cunningham Jr

\begin{abstract}
Aims-To describe the clinical course and treatment of Haemophilus influenzae associated scleritis.

Methods-Retrospective case series. Results-Three patients developed scleritis associated with ocular $H$ influenzae infection. Past medical history, review of systems, and laboratory testing for underlying collagen vascular disorders were negative in two patients. One patient had arthritis associated with an antinuclear antibody titre of 1:160 and a Westergren erythrocyte sedimentation rate of $83 \mathrm{~mm}$ in the first hour. Each patient had ocular surgery more than 6 months before developing scleritis. Two had cataract extraction and one had strabismus surgery. Nodular abscesses associated with areas of scleral necrosis were present in each case. Culture of these abscesses revealed $H$ influenzae in all patients. Treatments included topical, subconjunctival, and systemic antibiotics. Scleral inflammation resolved and visual acuity improved in each case.

Conclusion-H influenzae infection may be associated with scleritis. Accurate diagnosis and treatment may preserve ocular integrity and good visual acuity. (Br F Ophthalmol 1999;83:410-413)
\end{abstract}

The Francis I Proctor Foundation J P Whitcher E T Cunningham Jr

Department of Ophthalmology, UCSF, School of Medicine, San Francisco, California S O Sykes J P Whitcher E T Cunningham Jr

Department of Ophthalmology, The Cleveland Clinic

Foundation, Cleveland, Ohio

C Riemann

D M Meisler

C Y Lowder

Department of Ophthalmology, University of Puerto

Rico School of Medicine, San Juan, PR 00927

C I Santos

Correspondence to: Dr Emmett T Cunningham Jr, The Pearl and Samuel J Kimura Ocular Immunology Laboratory, The Francis I Proctor Foundation, UCSF, School of Medicine, San Francisco, CA 94143-0944, USA.

Accepted for publication 22 October 1998
Necrotising scleritis is a serious and destructive ocular disorder. ${ }^{12}$ Many cases can be related to a potentially life threatening systemic autoimmune disorder, most frequently rheumatoid arthritis..$^{2-4}$ Infection as a cause of scleritis is, in contrast, uncommon, accounting for approximately $5-15 \%$ of cases in previously reported series. ${ }^{5-7}$ The most commonly identified organisms have included Pseudomonas aeruginosa, ${ }^{8-14}$ Streptococcus pneumoniae, ${ }^{12-16}$ staphylococcal species, ${ }^{713141617}$ and varicella zoster virus. ${ }^{710} 18$ We present three patients with scleral inflammation and necrosis associated with ocular H influenzae infection.

\section{Materials and methods}

The clinical and photographic records of three patients with $H$ influenzae associated scleritis were reviewed retrospectively. The presentation, approach to diagnosis, and response to therapy were summarised.

\section{Results}

CASE 1

A 78 year old woman was referred for evaluation and treatment of right eye pain. Past ocular history was significant for cataract surgery more than 3 years before presentation. Past medical history included arthritis of unknown type and
Alzheimer's dementia. Eye examination revealed a best corrected visual acuity of $6 / 60$ in the right eye and 6/12 in the left eye. Slit lamp examination revealed bilateral superior scleral thinning with a sector of active scleritis in the right eye. Other findings included bilateral posterior capsule opacification and macular retinal pigment epithelium irregularities, worse on the left. Laboratory testing revealed an antinuclear antibody (ANA) titre of 1:160 and a Westergren elevated erythrocyte sedimentation rate (ESR) of $83 \mathrm{~mm}$ in the first hour. Rheumatoid factor (RF) was negative. The patient was diagnosed with idiopathic scleritis, and treated with oral methotrexate, $10 \mathrm{mg} /$ week, and hourly prednisolone acetate, $1 \%$.

After 10 weeks of therapy the superior scleritis resolved but a new area of nodular scleritis developed inferotemporally. The methotrexate was increased to $12.5 \mathrm{mg} /$ week and oral prednisone, $40 \mathrm{mg} /$ day, was added. Within 3 weeks the area of inferotemporal scleritis developed into a small nodular abscess with an intact overlying epithelium (Fig 1A). The abscess was incised and cultures of the expressed, purulent material grew H influenzae sensitive to third generation cephalosporins. The patient was treated with intravenous ceftriaxone, $1 \mathrm{~g} /$ day, and fortified, topical cefuroxime, $50 \mathrm{mg} / \mathrm{ml}$, administered hourly. Systemic evaluation, including blood and urine cultures, and chest $x$ ray were negative. The scleral abscess worsened initially, prompting an increase in the intravenous ceftriaxone to 2000 $\mathrm{mg}$ /day. Supplemental subconjunctival ceftazidime injections (100 mg/0.5 ml) were also given for 3 consecutive days. The scleritis began to improve on the third hospital day and the patient was discharged on fortified topical cefuroxime drops and oral cefuroxime $500 \mathrm{mg}$ given orally twice daily. Subconjunctival injections of ceftazidime were given for three additional days after discharge. Complete resolution of inflammation was observed 3 weeks after onset of antibiotic therapy (Fig 1B). Visual acuity of the right eye following laser posterior capsulotomy 6 weeks after the infection improved to $6 / 18$. The eye remained free of inflammation for 7 months of follow up.

\section{CASE 2}

A 74 year old woman with diabetes mellitus and osteoarthritis developed a red, irritated right eye 10 months after cataract surgery. Her local ophthalmologist found and removed a loose suture from the superior limbal wound. Eye symptoms worsened and the patient was referred for evaluation and treatment. Examination of the left eye was unremarkable. Examination of the right eye revealed a best corrected visual acuity of $6 / 12$ and a large area 


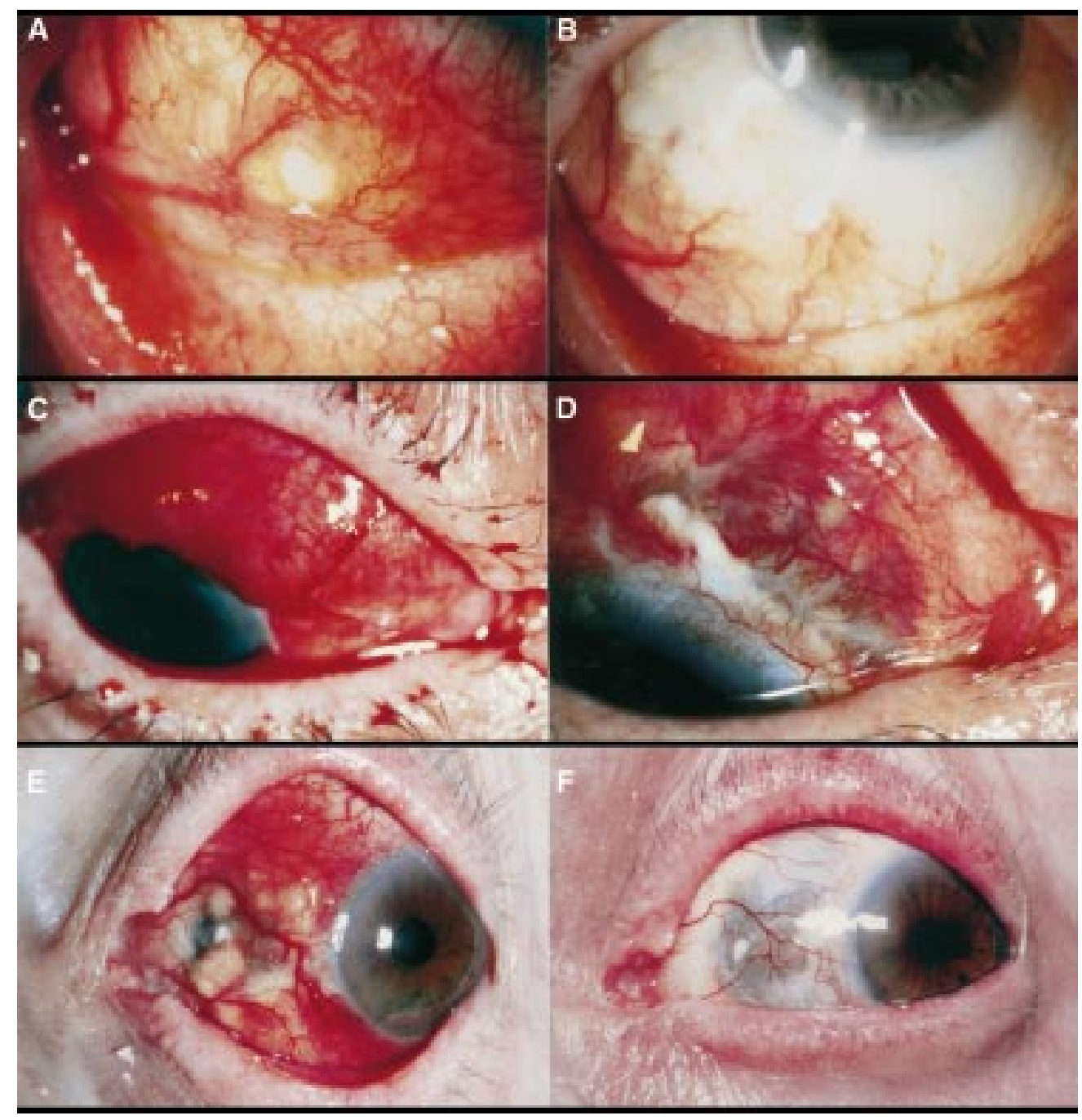

Figure 1 (A) Case 1. Inferotemporal scleral injection with a relatively avascular nodular abscess. (B) Case 1. Appearance of the same area following 3 weeks of treatment shows complete resolution of the scleritis and nodular abscess. (C) Case 2. Diffusely injected superior sclera with a large, vascularised abscess in an area of recent suture removal. (D) Case 2. Appearance of the same area after 4 weeks of therapy and multiple debridements shows residual thinning and an early subconjunctival fibrovascular scar. (E) Case 3. Inflammatory thinning of the sclera with multiple, adjacent nodular abscesses near the insertion of the left medial rectus muscle. (F) Case 3. Appearance of the same area 6 months after therapy shows complete resolution of the inflammation and nodules. The area of scleral thinning has expanded.

of superior, necrotising scleritis associated with subconjunctival haemorrhage and a nodule at the site of recent suture removal. The nodule was surgically debrided, cultures were taken, and subconjunctival vancomycin $(25 \mathrm{mg} / 0.5$ $\mathrm{ml})$ and gentamicin $(20 \mathrm{mg} / 0.5 \mathrm{ml})$ were injected. Topical fluorometholone, $0.25 \%$, one drop four times per day, oral prednisone, 50 $\mathrm{mg} /$ day, and oral indomethacin, $25 \mathrm{mg} /$ day, were also started. Bacterial and fungal cultures were initially negative.

The scleritis improved initially but by 2 weeks the area of scleritis began to worsen and a large, superior scleral abscess formed requiring repeat surgical debridement (Fig 1C). Additional subconjunctival injections of vancomycin and gentamicin were given. Hourly, topical, fortified cefazolin $(50 \mathrm{mg} / \mathrm{ml})$ and gentamicin $(9 \mathrm{mg} / \mathrm{ml})$ were also initiated. Repeat cultures taken at the time of debridement of the abscess grew $H$ influenzae sensitive to penicillins, cephalosporins, and aminoglycosides. Oral ampicillin (500 mg/day) was added. The abscess was drained again and subconjunctival injections were given a third time. The abscess slowly resolved over 4 weeks leaving scleral thinning and a subconjunctival fibrovascular scar (Fig 1D). Visual acuity on the right was $6 / 6$ at last follow up.

\section{CASE 3}

A healthy 86 year old woman with a history of strabismus surgery 10 years before presentation complained of pain and redness of the left eye of 2 weeks' duration. Examination by her local ophthalmologist revealed an area of scleral necrosis near the insertion of left medial rectus muscle. The patient was treated with oral prednisone, $60 \mathrm{mg} /$ day, for 1 week but the scleritis worsened and the patient was referred for evaluation and treatment. Examination of the left eye revealed a best corrected visual acuity of $6 / 30$ and an area of scleral inflammation and thinning with adjacent nodular abscesses near the insertion of the left medial rectus muscle (Fig 1E). The remainder of the 
eye examination was notable for moderate nuclear sclerotic cataract in each eye. The conjunctiva was cultured, and topical polymixin B trimethoprim, administered every 2 hours, was added to the oral prednisone. Laboratory testing revealed a normal ESR and chest $x$ ray, negative ANA, RF, and antineutrophil cytoplasmic antibody (ANCA) titres, negative skin testing with purified protein derivative (PPD), and negative syphilis serologies. Conjunctival cultures grew $H$ influenzae sensitive to ciprofloxacin, ceftazidime, and chloramphenicol.

One week later the scleritis worsened and a nodular abscess was debrided and cultured. Cultures of the debrided material again grew $H$ influenzae. Topical polymixin B trimethoprim was continued, but prednisone was quickly tapered because of worsening confusion and disorientation. Postoperative subconjunctival ceftazidime $(100 \mathrm{mg} / 0.5 \mathrm{ml})$ was administered and topical $(0.3 \%$, every 2 hours) and oral (150 mg, twice daily) ciprofloxacin were initiated. The scleritis improved rapidly, with eventual resolution of both the inflammation and nodular abscesses (Fig 1F). Visual acuity 9 months after the presentation was $6 / 12$, consistent with the amount of nuclear sclerotic cataract.

\section{Discussion}

Infectious scleritis is a serious but uncommon ocular disorder. The three cases of $H$ influenzae associated scleritis we described shared several distinguishing features, including the presence of nodular abscesses and necrosis, worsening on treatment with corticosteroids, and response to appropriate antibiotic therapy after identification of the infectious organism. Good visual recovery occurred in each case.

Haemophilus influenzae is a small Gram negative coccobacillus that infects humans exclusively. Up to $80 \%$ of people harbour the organism in the upper respiratory tract but it can be found on other mucosal surfaces as well, including the genital tract and conjunctiva. ${ }^{19}$ As an ocular pathogen, $H$ influenzae is a well recognised cause of both conjunctivitis in infants and children, ${ }^{20}$ and of bleb associated endophthalmitis. ${ }^{21-24}$ The reason for $H$ influenzae's tendency to cause conjunctivitis and bleb associated infections is unknown, although it is tempting to speculate that this may be related to its known tropism for mucosal surfaces, where replicating organisms have been found in both epithelial cells and macrophages in subepithelial layers. ${ }^{25}$ This phenomenon is termed "epithelial parasitism", and may have played a role in the pathogenesis of the deeper, necrotising infections observed in our three patients with $H$ influenzae associated scleritis.

Infectious scleritis usually occurs by secondary spread from an adjacent corneal ulcer..$^{11-13}$ In the rare case in which the sclera is infected primarily, an underlying risk factor is usually present, ${ }^{67}$ including prior scleritis, ${ }^{15}$ recent or remote ocular surgery, ${ }^{72}$ recent suture removal, ${ }^{13}$ ocular irradiation or antimetabolite use after pterygium surgery, ${ }^{10}{ }^{14}{ }^{16}$ use of topical corticosteroid preparations, ${ }^{11} 13$ or a systemic infection. ${ }^{16}{ }^{18}{ }^{26}$ Our three patients with $\mathrm{Hinflu}$ enzae associated scleritis all had prior ocular surgery, one had recent suture removal, and one had prior scleritis. In addition, one of our patients had serological evidence of a systemic inflammatory disorder, including an elevated ANA titre and a raised ESR, and one had a history of diabetes mellitus, conditions that may have predisposed these two patients to infection.

Prompt evaluation and treatment is essential for successful management of infectious scleritis. Diagnostic evaluation should include a thorough history, physical examination, and directed laboratory testing to rule out a predisposing systemic illness, concurrent systemic infection, or underlying autoimmune disorder. Material for cultures and Gram stain should be obtained from the involved sclera. Once recognised, the management of bacterial scleritis consists of intensive topical and subconjunctival antibiotics guided by sensitivity testing of the identified organisms. Systemic antibiotic therapy is usually indicated, ${ }^{67}$ particularly for $H$ influenzae where nasopharyngeal colonisation is common. ${ }^{25}$ Corticosteroid therapy for infectious scleritis is somewhat controversial, with most authors currently recommending cautious use, and only in the setting of appropriate antibiotic therapy. ${ }^{6} 7113$ Excisional biopsy, conjunctival resection, cryotherapy, and lamellar dissections with scleral grafting may also be necessary for severe or worsening infections. ${ }^{913}$

In summary, infection by $H$ influenzae should be considered in any patient with scleritis, particularly when the aforementioned risk factors are present and when observed in association with nodular abscesses and necrosis. Our patients with $H$ influenzae associated scleritis all did well with directed and aggressive antibiotic treatment once the causative organism was identified.

This work was supported by a career development award from Research to Prevent Blindness, Inc (ETC).

1 de la Maza MS, Jabbur NS, Foster CS. Severity of scleritis and episcleritis. Ophthalmology 1994;101:389-96.

2 Watson PG, Hazleman, BL. The sclera and systemic disorders. Philadelphia: WB Saunders, 1976.

3 de la Maza MS, Foster CS, Jabbur NS. Scleritis associated with systemic vasculitic diseases. Ophthalmology 1995;102: 687-92.

4 Foster CS, Forstot SL, Wilson LA. Mortality rate in rheumatoid arthritis patients developing necrotizing scleritis or peripheral ulcerative keratitis: effects of systemic immunosuppression. Ophthalmology 1984;91:1253-63.

5 Tuft SJ, Watson PG. Progression of scleral disease. Ophthalmology 1991;98:467-71.

6 Raizman MB. Microbial scleritis. In: Pepose JS, Holland GN, Wilhelmus KR, eds. Ocular infection and immunity. St

7 Foster CS, de la Maza MS. Infectious scleritis: the Massachussetts Eye and Ear Infirmary experience. In: Foster CS, de la Maza MS, eds. The sclera. New York: Springer-Verlag, Chapter 7, 1994:242-77.

8 Hehm CJ, Holland GN, Webster RG Jr, et al. Combination intravenous ceftazidime and aminoglycoside in the treatment of pseudomonal scleritis. Ophthalmology 1997;104: $838-43$.

9 Eiferman RA. Cryotherapy of Pseudomonas keratitis and scleritis. Arch Ophthalmol 1979;97:1637-9.

10 Rao NA, Marak GE, Hidayat AA. Necrotizing scleritis. A clinico-pathologic study of 41 cases. Ophthalmology 1985; 92:1542-9.

11 Alfonso E, Kenyon KR, Ormerod LD, et al. Pseudomonas corneoscleritis. Am $\mathcal{7}$ Ophthalmol 1987;103:90-8.

2 Farell PLR, Smith RE. Bacterial corneoscleritis complicating pterygium excision. Am f Ophthalmol 1989;107:515-7. 13 Reynolds MG, Alfonso E. Treatment of infectious scleritis and keratoscleritis. Am $\mathcal{F}$ Ophthalmol 1991;112:543-7. 
14 Moriarty AP, Crawford GJ, McAllister IL, et al. Severe corneoscleral infection. A complication of beta irradiation scleral necrosis following pterygium excision. Arch Ophthalmol 1993;111:947-51

15 Altman AJ, Cohen EJ, Berger ST, et al. Scleritis and Streptococcus pneumoniae. Cornea 1991;10:341-5.

16 Maskin SL. Infectious scleritis after a diabetic foot ulcer. $A m$ f Ophthalmol 1993;115:254-5.

17 de la Maza MS, Hemady RK, Foster CS. Infectious scleritis: report of four cases. Doc Ophthalmol 1993;83:33-41.

18 Hemady R, de la Maza MS, Raizman MB, et al. Six cases of slceritis associated with systemic infection. Am 7 Ophthalmol 1992;114:55-62.

19 Moxon ER. Haemophilus influenzae. In: Mandel GL, Douglas RG Jr, Bennett JE, eds. Principles and practice of infectious disease. 3rd ed. New York: Churchill Livingstone, 1990:1722.

20 Krohn JA, Hillier SL, Bell TA, et al and the Eye Prophylaxis Study Group. The bacterial etiology of conjunctivitis in Study Group. The bacterial etiology of conju
21 Mandelbaum S, Forster RK, Gelender H, et al. Late onset endophthalmitis associated with filtering blebs. Ophthalmology 1985;92:964-72.

22 Ciulla TA, Beck AD, Topping TM, et al. Blebitis, early endophthalmitis, and late endophthalmitis after glaucomafiltering surgery. Ophthalmology 1997;104:986-95.

23 Greenfield DS, Suner IJ, Miller MP, et al. Endophthalmitis after filtering surgery with mitomycin. Arch Ophthalmol 1996;114:943-9.

24 Irvine WD, Flynn HW Jr, Miller D, et al. Endophthalmitis caused by Gram-negative organisms. Arch Ophthalmol 1992;110:1450-4.

25 Forsgren J, Samuelson A, Ahlin A, J et al. Haemophilus influenzae resides and multiplies intracellularly in human adenoid tissue as demonstrated by in situ hybridization and bacterial viability assay. Infect Immun 1994;62:673-9.

26 Hwang DG. Systemic antibiotic therapy for relapsing Haemophilus influenzae conjunctivitis. Am F Ophthalmol 1993; 115:814-15. 